\title{
Is Hepatitis C Infection Associated With a Higher Risk of Complications After Total Shoulder Arthroplasty?
}

\author{
Jourdan M. Cancienne MD, Ian J. Dempsey MD, MBA, Russell E. Holzgrefe MD, \\ Stephen F. Brockmeier MD, Brian C. Werner MD
}

Received: 24 March 2016/Accepted: 7 July 2016/Published online: 22 July 2016

(C) The Association of Bone and Joint Surgeons $\mathbb{R} 2016$

\begin{abstract}
Background Despite recent advances in the treatment of hepatitis $\mathrm{C}$, it is estimated that nearly 4 million Americans have a chronic form of the disease. Although research in lower-extremity arthroplasty suggests patients with hepatitis $\mathrm{C}$ are at risk for increased complications, including postoperative bleeding, acute postoperative infection, and general medical complications, no similar studies have investigated this question in patients undergoing total shoulder arthroplasty (TSA).

Questions/purposes We asked whether there is an increased risk of postoperative complications after TSA among patients who have hepatitis $\mathrm{C}$, and if so, what complications in particular seem more likely to occur in this population?

Methods Patients who underwent TSA, including anatomic or reverse TSA, were identified in the PearlDiver database using ICD-9 procedure codes. This is a for-fee insurance patient-records database that contains more than
\end{abstract}

Each author certifies that he or she, or a member of his or her immediate family, has no funding or commercial associations (eg, consultancies, stock ownership, equity interest, patent/licensing arrangements, etc) that might pose a conflict of interest in connection with the submitted article.

All ICMJE Conflict of Interest Forms for authors and Clinical Orthopaedics and Related Research ${ }^{\mathbb{R}}$ editors and board members are on file with the publication and can be viewed on request.

Each author certifies that his or her institution approved or waived approval for the human protocol for this investigation and that all investigations were conducted in conformity with ethical principles of research.

J. M. Cancienne, I. J. Dempsey, R. E. Holzgrefe,

S. F. Brockmeier, B. C. Werner $(\bowtie)$

Department of Orthopaedic Surgery, University of Virginia, PO

Box 800136, Charlottesville, VA 22908, USA

e-mail: bcw4x@virginia.edu
100 million individual patient records from 2005 to 2012 . The Medicare data in the database are the complete $100 \%$ Medicare Standard Analytical File indexed to allow for patient tracking with time. Patients with hepatitis $\mathrm{C}$ who underwent shoulder arthroplasty then were identified using ICD-9 codes. Patients with hepatitis B coinfection or HIV were excluded. A control cohort of patients without hepatitis $\mathrm{C}$ who underwent TSA was created and matched to the study cohort based on age, sex, obesity, and diabetes mellitus. A total of 1466 patients with hepatitis $\mathrm{C}$ and 21,502 control patients were included. The two cohorts were statistically similar in terms of sex $(53 \%$ females in study and control groups), age (nearly $1 / 2$ of each cohort younger than 65 years), obesity (approximately 17\% of each cohort were obese), diabetes (approximately $40 \%$ of each cohort had diabetes), and followup of each cohort occurred throughout the length of the database from 2005 to 2012. Postoperative complications were assessed using ICD-9 and Current Procedural Terminology codes and compared between cohorts.

Results Patients with hepatitis C, when compared with matched control subjects, had greater odds of infection within 3 months (odds ratio [OR], 1.7; 95\% CI, 1.1-2.6; p $=0.015), 6$ months $(\mathrm{OR}, 1.7 ; \mathrm{CI}, 1.3-2.4 ; \mathrm{p}=0.001)$, and 1 year (OR, 2.1; CI, 1.7-2.7; $<<0.001)$; revision TSA within 1 year $(\mathrm{OR}, 1.5 ; \mathrm{CI}, 1.1-2.9 ; \mathrm{p}=0.008)$ and 2 years $(\mathrm{OR}$, 1.6; CI, 1.2-2.0; $\mathrm{p}=0.001)$, dislocation within 1 year $(\mathrm{OR}$, 1.6; CI, 1.2-2.2; $\mathrm{p}<0.001)$; postoperative fracture within 1 year (OR, 1.8; CI, 1.2-2.6; $\mathrm{p}=0.002)$; systemic or medical complications within 3 months (OR, 1.3; CI, 1.0-1.6; $\mathrm{p}=$ 0.022 ); and blood transfusion within 3 months (OR, 1.7; CI, 1.4-1.9; $\mathrm{p}<0.001)$.

Conclusions Hepatitis $\mathrm{C}$ is associated with an increased risk for complications after TSA, including infection, dislocation, fracture, revision TSA, systemic complications, 
and blood transfusion compared with matched control subjects. Although this study is able to identify increased odds of complications in patients with hepatitis $\mathrm{C}$, the mechanism by which these occur is likely not solely related to the virus, and is more likely related to a higher degree of case complexity in addition to other postoperative socioeconomic factors.

Level of Evidence Level III, therapeutic study.

\section{Introduction}

Although the prevalence of hepatitis $\mathrm{C}$ infection among patients undergoing orthopaedic surgery has been estimated to be from $3 \%$ to $8 \%$, studies in the setting of lowerextremity arthroplasty have reported an increase in the number of patients with hepatitis $\mathrm{C}$ undergoing lower-extremity total joint arthroplasty during the last decade [4, 8]. Furthermore, these studies raise concerns regarding the apparent increase in postoperative complications in patients with hepatitis $\mathrm{C}$ compared with those without the virus. Recent lower-extremity arthroplasty literature has shown a significant increase in the odds of acute postoperative infection, mechanical complications, allogenic blood transfusion, and general medical and systemic complications [3, 8]. This is likely attributable to the complex pathophysiology of hepatitis $\mathrm{C}$ infection and its association with several extrahepatic manifestations that likely contribute to the described increase in perioperative complications including hepatitis, membranoproliferative glomerulonephritis, leukocytoclastic vasculitis, and thrombocytopenia $[1,10,12,14,16,17,22]$.

During the past decade, the number of total shoulder arthroplasties (TSAs) performed in the United States has increased dramatically, with more than 50,000 currently being performed per year. In addition, annual rates of TSA are increasing at a higher rate than lower-extremity arthroplasties, making TSA one of the most commonly performed elective procedures in the elderly population today $[5,9,11]$. Although research in lower-extremity arthroplasty suggests patients with hepatitis $\mathrm{C}$ are at risk for increased complications, no similar studies have investigated this question in patients undergoing TSA. Although multiple medical comorbidities and conditions have been shown to adversely affect patient outcomes after TSA, there has been no literature to date, to our knowledge, investigating the subset of patients with hepatitis $\mathrm{C}$ deciding to undergo elective TSA [2, 6, 13, 20, 21, 23]. Recent medical treatments for hepatitis $\mathrm{C}$ have shown reliable and high cure rates [19]. However, these treatments remain exceedingly expensive and therefore widespread access is limited, and there is a large subset of patients with chronic hepatitis C. Given the increased focus on reducing the social and financial burden of complications after total joint arthroplasty, it is important to evaluate any association between hepatitis $\mathrm{C}$ and increased postoperative complications after TSA.

We therefore asked: Is there an increased risk of postoperative complications after TSA among patients who have hepatitis $\mathrm{C}$, and if so, which complications particularly seem more likely to occur in this population?

\section{Patients and Methods}

The PearlDiver patient records database (http://www. pearldiverinc.com/; PearlDiver Technologies Inc, West Conshohocken, PA, USA), a for-fee insurance-based patient-records database, was used for our study. An exemption was issued by the authors' institutional review board, because the study involved only deidentified information from a publicly available administrative database. The database consists of several separate private insurers and a Medicare database with procedural volumes and patient demographics for patients with ICD-9 diagnoses and procedures or Current Procedural Terminology (CPT) codes. The data for the study were derived from the Medicare database in PearlDiver, which contains more than 100 million individual patients' records from 2005 to 2012.

We sought to define a study population of patients with hepatitis $\mathrm{C}$ who underwent TSA before widespread use of antiviral therapies. The database was first queried for all patients who underwent TSA or reverse TSA using the ICD-9 procedure codes 81.80 and 81.88 . Patients with hepatitis $\mathrm{C}$ who underwent shoulder arthroplasty then were identified using the ICD-9 diagnostic codes 070.54 and 070.70. The indications for and breakdown of patients in each cohort who underwent TSA or reverse TSA then were determined. Patients coinfected with hepatitis B and HIV were excluded from the final study cohort.

After application of these inclusion and exclusion criteria, a control cohort of patients without hepatitis $\mathrm{C}$ who underwent shoulder arthroplasty was created and matched to the study cohort based on age, sex, obesity, and diabetes mellitus. Although the database allows matching of demographics and comorbidities, there are several significant confounding variables that are not identifiable in the database that must be considered. We suspect that patients with hepatitis $\mathrm{C}$ might be more likely to use intravenous drugs and alcohol, or have different socioeconomic backgrounds, such as a higher incidence of homelessness and lack insurance, than our control cohort. These, among many other factors, might result in these patients presenting later for care or having more advanced deformities, resulting in a higher level of case complexity, leading to 
Table 1. Comparison of 1466 patients with hepatitis $C$ and 21,502 matched control subjects

\begin{tabular}{llcc}
\hline Variable & $\begin{array}{l}\text { Patients with hepatitis C } \\
\text { Number }(\%)\end{array}$ & $\begin{array}{l}\text { Control subjects } \\
\text { Number }(\%)\end{array}$ & $11,454(53 \%)$ \\
\hline Female & $781(53 \%)$ & & \\
Age of patients & & $9390(44 \%)$ & 0.981 \\
$<65$ years & $639(44 \%)$ & $3776(18 \%)$ & 0.973 \\
$65-69$ years & $254(17 \%)$ & $3520(16 \%)$ & 0.847 \\
$70-74$ years & $240(16 \%)$ & $2928(14 \%)$ & 0.971 \\
$75-79$ years & $202(14 \%)$ & $1347(6 \%)$ & 0.892 \\
$80-84$ years & $93(6 \%)$ & $541(3 \%)$ & 0.948 \\
$\geq 85$ years & $38(3 \%)$ & $3849(18 \%)$ & 0.925 \\
Comorbidities & & $8067(38 \%)$ \\
Obesity $\left(\right.$ BMI $\left.\geq 30 \mathrm{~kg} / \mathrm{m}^{2}\right)$ & $249(17 \%)$ & 0.395 \\
Diabetes mellitus & $586(40 \%)$ & 064 \\
\hline
\end{tabular}

increased odds of postoperative complications. These variables are unable to be accounted for in our analysis and must be considered.

Postoperative complications within 90 days, 6 months, 1 year, and 2 years after shoulder arthroplasty then were identified in the database for the study and matched control cohorts.

Followup in the database occurred throughout the length of the study period, from 2005 to 2012, thus there is no differential followup between the study and control cohorts. Infection within 3 months, 6 months, and 1 year postoperatively was assessed using the following ICD-9 diagnostic codes: 99.851, 99.859, 71.101, 71.181, 71.191, 99.666, 99.669, 99.660, 99.831, and 99.667 in association with the following CPT codes: 10180, 20005, 23030, 23031, 23040, and 23035. Additional CPT codes specific for removal of shoulder implants became available in 2014, therefore our study captures data only from 2005 to 2012. Revision TSA within 1 year and 2 years postoperatively was assessed using the ICD-9 procedure code 81.97. Dislocation, implant loosening, and postoperative fracture within 1 year were assessed using ICD-9 codes for each. Finally, systemic complications and allogeneic blood transfusion odds ratios were determined for each cohort using ICD-9 codes for each complication.

\section{Statistical Analysis}

Statistical comparisons of the cohort demographics and odds of postoperative complications were completed using Pearson chi-square analysis. Odds ratios (ORs) were calculated with respective $95 \%$ CIs. For all statistical comparisons, a probability less than 0.05 was considered significant. SPSS Version 23.0 for Macintosh (IBM, Armonk, NY, USA) was used for all statistical calculations.
There were no statistically significant differences present in the measured demographics or comorbidities between the study and control cohorts, indicating successful matching (Table 1). Slightly more than $1 / 2$ of the patients in each cohort were female, and the majority of the patients in each cohort was younger than 65 years. As is common with hepatitis $C$, nearly $1 / 2$ of the study cohort had concomitant diabetes mellitus; however, to prevent confounding, there was no difference in the number of patients with diabetes in our control cohort. Finally, approximately $17 \%$ of each cohort had a BMI greater than or equal to 30 $\mathrm{kg} / \mathrm{m}^{2}$.

\section{Results}

A total of 22,968 unique patients who underwent TSA or revision TSA met inclusion and exclusion criteria and were included in the study. This included 1466 patients with hepatitis $\mathrm{C}$ and 21,502 matched control subjects. Three hundred patients in the study cohort underwent reverse TSA compared with 4100 in the matched control cohort. The primary indication for shoulder arthroplasty in the study cohort included osteoarthritis $(81 \%)$, rotator cuff arthropathy $(24 \%)$, rheumatoid arthritis $(8 \%)$, avascular necrosis $(5 \%)$, malunion or nonunion of a fracture $(4 \%)$, and acute fracture $(4 \%)$. Some patients had more than one diagnostic indication for arthroplasty. There were no significant differences in the indications for shoulder arthroplasty in the control cohort compared with the study cohort. Comparing patients with hepatitis $\mathrm{C}$ with control subjects, there is greater odds of postoperative infection within 3 months (25 of 1466 [2\%] versus 215 of 21,502 [1\%]; OR, 1.7; 95\% CI, 1.1-2.6; $\mathrm{p}=0.015), 6$ months (45 [3\%] versus 384 [2\%]; OR, 1.7; 95\% CI, 1.3-2.4; p = $0.001)$, and 1 year (88 [6\%] versus 630 [3\%]; OR, 2.1; 95\% 
Table 2. Comparison of postoperative complications in patients with hepatitis $\mathrm{C}$ and control subjects

\begin{tabular}{lccc}
\hline Complication & $\begin{array}{l}1466 \text { patients with hepatitis C } \\
\text { Number }(\%)\end{array}$ & $\begin{array}{l}21,502 \text { control subjects } \\
\text { Number }(\%)\end{array}$ & OR (95\% CI) \\
\hline Infection & & & \\
Within 3 months & $25(2 \%)$ & $215(1 \%)$ & $1.7(1.1-2.6)$ \\
Within 6 months & $45(3 \%)$ & $384(2 \%)$ & $1.7(1.3-2.4)$ \\
Within 1 year & $88(6 \%)$ & $630(3 \%)$ & $2.1(1.7-2.7)$ \\
Revision TSA & & & 0.015 \\
Within 1 year & $50(3 \%)$ & $492(2 \%)$ & $1.5(1.1-2.0)$ \\
Within 2 years & $70(5 \%)$ & $669(3 \%)$ & $1.6(1.2-2.0)$ \\
Dislocation (1 year) & $56(4 \%)$ & $510(2 \%)$ & $1.6(1.2-2.2)$ \\
Stiffness (1 year) & $126(9 \%)$ & $1665(8 \%)$ & $1.1(0.9-1.4)$ \\
Loosening (1 year) & $21(1 \%)$ & $202(1 \%)$ & 0.008 \\
Fracture (1 year) & $32(2 \%)$ & $263(1 \%)$ & 0.001 \\
Systemic (3 months) & $87(6 \%)$ & $988(5 \%)$ & 0.2601 \\
Transfusion (3 months) & $192(13 \%)$ & $1799(8 \%)$ & $1.0-2.4)$ \\
\hline
\end{tabular}

TSA = total shoulder arthroplasty; OR = odds ratio.

CI, 1.7-2.7; p < 0.001) (Table 2), and the odds of revision TSA also was higher in patients with hepatitis $\mathrm{C}$ at 1 year (50 [3\%] versus 492 [2\%]; OR, 1.5; 95\% CI, 1.1-2.0; $p=$ $0.008)$ and 2 years $(70[5 \%]$ versus $669[3 \%] ;$ OR, 1.6; $95 \%$ CI, 1.2-2.0; $\mathrm{p}=0.001)$. Likewise, the odds of dislocation (56 [4\%] versus 510 [2\%]; OR, 1.6; 95\% CI, 1.2$2.2 ; \mathrm{p}=0.001)$ and postoperative fracture (32 [2\%] versus 263 [1\%]; OR, 1.8; 95\% CI, 1.2-2.6; $\mathrm{p}=0.002$ ) within 1 year were greater in patients with hepatitis $\mathrm{C}$ compared with matched control subjects. Systemic complications (87 [6\%] versus 988 [5\%]; OR, 1.3; 95\% CI, 1.0-1.6; p = $0.022)$ and allogeneic blood transfusion (192 [13\%] versus 1799 [8\%]; OR, 1.7; 95\% CI, 1.4-1.9; $p<0.0001)$ within 3 months were higher in patients with hepatitis $\mathrm{C}$ compared with matched control subjects. There was no difference in the incidence of postoperative loosening (21 [1\%] versus 202 [1\%]; OR, $1.5 ; 95 \%$ CI, $1.0-2.4 ; \mathrm{p}=0.085$ ) within 1 year in patients with hepatitis $\mathrm{C}$ compared with matched control subjects.

\section{Discussion}

Risk stratification in patients undergoing TSA has become increasingly important to improve patient outcomes and decrease the social and financial burdens of postoperative complications after total joint arthroplasty. Although the association between a patient's hepatitis $C$ status and the increased risk of postoperative bleeding, acute postoperative infection, and general medical complications have been studied in patients undergoing lower-extremity arthroplasty $[3,8]$, no studies of which we are aware have investigated the subset of patients undergoing TSA with hepatitis C. Our study suggests that patients with hepatitis $\mathrm{C}$ are at an increased risk for complications after TSA, including infection, dislocation, fracture, revision TSA, systemic complications, and blood transfusion, compared with matched control subjects.

This study has several limitations. First, and most importantly, a limitation of all database studies is the inability to control for numerous confounding variables that are not identifiable in the database. As discussed in the Patients and Methods section, there are several patient factors that are unable to be searched for in the database. Some of these include the complex socioeconomic challenges that patients with hepatitis $\mathrm{C}$ face. Things such as intravenous drug abuse, homelessness, alcoholism, access to healthcare, and insurance likely affect the outcome of TSA in this population. However, these are intangible elements that cannot be accounted for or studied in the database. Thus, our results must be considered in this context, and we suggest that the effect of hepatitis $\mathrm{C}$ on outcomes after TSA is multifactorial, and perhaps should be viewed more as a marker for a higher level of case complexity leading to increased complications than a stand-alone risk factor. Second, the robustness of our analysis relies on the quality of the data and the accuracy that these procedures and complications are coded. Thus, miscoding and noncoding by physicians and coders are potential sources of error. A recent Centers for Medicare \& Medicaid Services improper payments report from 2012 cited an overall coding error rate of $1.3 \%$ [7]. Therefore, although this is a major potential limitation when using administrative databases such as PearlDiver, the overall coding error rate is low. Third, by using a database that draws from a Medicare sample, we attempt to present a 
large representative cross-section of the United States. However, these conclusions may not apply to a younger, private-payer population undergoing TSA. Fourth, because we report our results for two distinct cohorts, we are unable to perform a multivariate analysis of our results. Finally, the database used indexes data only from 2005 to 2012 . Thus each cohort is followed throughout this period. There are likely some patients who undergo TSA, with and without a diagnosis of hepatitis $\mathrm{C}$, who might have dropped out of the indexed data at the end of each time. Although this is likely a small number of patients, there are patients in the database for whom endpoints cannot be assessed.

Hepatitis $\mathrm{C}$ is associated with an increased risk for complications in these patients after TSA, including infection, dislocation, fracture, revision TSA, systemic complications, and blood transfusion compared with ageand sex-matched control subjects. These findings are consistent with those in the lower-extremity arthroplasty literature that also have showed an association between a patient's hepatitis $\mathrm{C}$ status and the increased risk of postoperative complications $[3,8]$. In a recent study by Issa et al. [8], the National Inpatient Sample database was used to identify more than 8044 patients who underwent either THA or TKA and compared them with a matched control cohort of patients without hepatitis $\mathrm{C}$ undergoing the same procedures. Compared with matched control subjects, patients with hepatitis $\mathrm{C}$ had a $30 \%$ increased risk of any complication, a $15 \%$ increased risk of a medical complication, and $78 \%$ increased risk of a surgical complication. These results were substantiated by a prior study by Pour et al. [18], who reviewed their institutional database and identified 72 patients with hepatitis $\mathrm{C}$ who underwent THA or TKA and compared them with a matched control cohort of patients without the virus. Patients with hepatitis $\mathrm{C}$ undergoing total joint arthroplasty had higher rates of surgical and mechanical complications, reoperation, and revision surgery. Finally, Best et al. [3] used the National Hospital Discharge Survey to compare 26,444 patients with hepatitis $\mathrm{C}$ undergoing THA or TKA with a control cohort of $8,336,882$ patients without the virus. They also reported higher rates of in-hospital complications, surgery-related complications, and general medical complications in patients with hepatitis $\mathrm{C}$ undergoing total joint arthroplasty. Although it would be expected that patients with hepatitis C undergoing TSA would have a similar increase in perioperative complications and poor outcomes, this relationship has not been studied until now to our knowledge. Although there is an impaired physiologic response to surgery that contributes to the increased systemic and prosthetic perioperative complications seen in our study cohorts, there also are multiple other confounding variables that are associated with the virus that lead to an overall higher case complexity, and thus complication rate. For instance, Pour et al. [18] proposed that the previously discussed immunemediated manifestations, even found in subclinical cases of hepatitis $\mathrm{C}$, lead to higher rates of wound healing problems, resulting in infection. In addition to the underlying pathophysiology, patients with hepatitis $\mathrm{C}$ may have limited access to followup and health care that could prevent and more appropriately treat infection. Our study shows higher rates of postoperative infection at all times in our hepatitis C cohort compared with matched control subjects, suggesting that the virus is also a risk factor for infectious complications after TSA. Furthermore, only one prior study reported on the implant longevity in patients with hepatitis C undergoing lower-extremity arthroplasty. Pour et al. [18] reported a higher rate of aseptic revision in patients with the virus compared with matched controls. We also found higher aseptic revision rates at all times in patients with hepatitis C compared with control subjects. Higher rates of aseptic revision may reflect a higher case complexity attributable to deformity or contracture that is present preoperatively resulting in poor implant positioning. While it is not in the scope of this study to determine the exact mechanism for this complication, it is unlikely that the virus accounts for greater odds of aseptic revision in this population, and more research is needed to determine what these other factors are. Although the study by Pour et al. [18] was insufficiently powered to detect a difference in the incidence of dislocation after THA, $8 \%$ of patients with hepatitis $\mathrm{C}$ had a periprosthetic dislocation compared with only $1.3 \%$ of their control cohort. Our patients with hepatitis $\mathrm{C}$ had an increased rate of postoperative dislocation. The underlying reason for increased instability after shoulder arthroplasty in this subset of patients is likely multifactorial, and a large component of this might be attributable to socioeconomic factors such as alcoholism and living conditions. Finally, similar to prior work in lower-extremity arthroplasty, we report higher perioperative transfusion rates in patients with hepatitis $\mathrm{C}$ undergoing TSA. It is known that even in the absence of cirrhosis, patients with hepatitis $\mathrm{C}$ have impaired platelet function resulting in thrombocytopenia, predisposing them to postoperative anemia requiring allogeneic blood transfusion $[14,16]$.

Hepatitis $\mathrm{C}$ in patients is associated with an increased risk for complications after TSA, including infection, dislocation, fracture, revision TSA, systemic complications, and blood transfusion compared with age- and sex-matched control subjects. It is important to consider the results of the study in the context of the design and limitations. Although we attempted to create matched cohorts to isolate the effects of hepatitis C on TSA, there are a myriad of medical and social variables that cannot be accounted for in the database. Thus, hepatitis $\mathrm{C}$ should be seen more as a surrogate for a greater degree of case complexity, 
intraoperatively and postoperatively, that results in the increased complications in our study patients. Given these findings, orthopaedic surgeons should be increasingly aware of this association, which should influence the shared decision-making process of undergoing TSA in patients with hepatitis C. Furthermore, this study shows a clear need for further research between the association of hepatitis $\mathrm{C}$ and increased complications in patients undergoing TSA. This research would quantify and consider the socioeconomic variables that might contribute to complications and identify strategies to prevent them. Furthermore, this research would seek to identify markers not dissimilar from A1c for diabetes, or echocardiograms for congestive heart failure, to predict perioperative surgical morbidity and mortality in patients with the virus. Finally, with the rapidly evolving antiviral therapies in modern medicine, it is important to determine the effects these treatments have on improving the physiologic state of the patient undergoing surgery [15]. Although these therapies have been successful in diminishing the viral load of the disease, it currently is unknown how this corresponds to the patient's overall surgical fitness.

\section{References}

1. Alter MJ, Margolis HS, Krawczynski K, Judson FN, Mares A, Alexander WJ, Hu PY, Miller JK, Gerber MA, Sampliner RE, et al. The natural history of community-acquired hepatitis $\mathrm{C}$ in the United States. The Sentinel Counties Chronic non-A, non-B Hepatitis Study Team. N Engl J Med. 1992;327:1899-1905.

2. Basques BA, Gardner EC, Toy JO, Golinvaux NS, Bohl DD, Grauer JN. Length of stay and readmission after total shoulder arthroplasty: an analysis of 1505 cases. Am J Orthop (Belle Mead NJ). 2015;44:E268-271.

3. Best MJ, Buller LT, Klika AK, Barsoum WK. Increase in perioperative complications following primary total hip and knee arthroplasty in patients with hepatitis C without cirrhosis. $J$ Arthroplasty. 2015;30:663-668.

4. Calore BL, Cheung RC, Giori NJ. Prevalence of hepatitis C virus infection in the veteran population undergoing total joint arthroplasty. J Arthroplasty. 2012;27:1772-1776.

5. Day JS, Lau E, Ong KL, Williams GR, Ramsey ML, Kurtz SM. Prevalence and projections of total shoulder and elbow arthroplasty in the United States to 2015. J Shoulder Elbow Surg. 2010;19:1115-1120.

6. Farng E, Zingmond D, Krenek L, Soohoo NF. Factors predicting complication rates after primary shoulder arthroplasty. J Shoulder Elbow Surg. 2011;20:557-563.
7. Centers for Medicare and Medicaid Services. Medicare Fee-forService 2012 Improper Payments Report Medicare Fee-for-Service 2012 Improper Payments Report. Available at: https://www.cms. gov/Research-Statistics-Data-and-Systems/Monitoring-Programs/ CERT/Downloads/MedicareFeeforService2012ImproperPayments Report.pdf. Accessed March 9, 2016.

8. Issa K, Boylan MR, Naziri Q, Perfetti DC, Maheshwari AV, Mont MA. The impact of hepatitis C on short-term outcomes of total joint arthroplasty. J Bone Joint Surg Am. 2015;97:19521957.

9. Jain NB, Higgins LD, Guller U, Pietrobon R, Katz JN. Trends in the epidemiology of total shoulder arthroplasty in the United States from 1990-2000. Arthritis Rheum. 2006;55:591-597.

10. Johnson RJ, Gretch DR, Yamabe H, Hart J, Bacchi CE, Hartwell P, Couser WG, Corey L, Wener MH, Alpers CE. Membranoproliferative glomerulonephritis associated with hepatitis $\mathrm{C}$ virus infection. N Engl J Med. 1993;328:465-470.

11. Kim SH, Wise BL, Zhang Y, Szabo RM. Increasing incidence of shoulder arthroplasty in the United States. J Bone Joint Surg Am. 2011;93:2249-2254.

12. Lauer GM, Walker BD. Hepatitis $\mathrm{C}$ virus infection. $N$ Engl $J$ Med. 2001;345:41-52.

13. Matsen FA, Li N, Gao H, Yuan S, Russ SM, Sampson PD. Factors affecting length of stay, readmission, and revision after shoulder arthroplasty: a population-based study. J Bone Joint Surg Am. 2015;97:1255-1263.

14. Mayo MJ. Extrahepatic manifestations of hepatitis C infection. Am J Med Sci. 2003;325:135-148.

15. Nyalakonda H, Utay NS. A new era of therapy for hepatitis C virus infection. Curr Opin Infect Dis. 2015;28:471-478.

16. Olariu M, Olariu C, Olteanu D. Thrombocytopenia in chronic hepatitis C. J Gastrointestin Liver Dis. 2010;19:381-385.

17. Palekar NA, Harrison SA. Extrahepatic manifestations of hepatitis C. South Med J. 2005;98:1019-1023.

18. Pour AE, Matar WY, Jafari SM, Purtill JJ, Austin MS, Parvizi J. Total joint arthroplasty in patients with hepatitis C. J Bone Joint Surg Am. 2011;93:1448-1454.

19. Rosen HR. Clinical practice: chronic hepatitis C infection. $N$ Engl J Med. 2011;364:2429-2438.

20. Schairer WW, Zhang AL, Feeley BT. Hospital readmissions after primary shoulder arthroplasty. $J$ Shoulder Elbow Surg. 2014;23:1349-1355.

21. Streubel PN, Simone JP, Sperling JW, Cofield R. Thirty and ninety-day reoperation rates after shoulder arthroplasty. $J$ Bone Joint Surg Am. 2014;96:e17.

22. Wang CS, Yao WJ, Wang ST, Chang TT, Chou P. Strong association of hepatitis $\mathrm{C}$ virus (HCV) infection and thrombocytopenia: implications from a survey of a community with hyperendemic HCV infection. Clin Infect Dis. 2004;39:790796.

23. Waterman BR, Dunn JC, Bader J, Urrea L, Schoenfeld AJ, Belmont PJ. Thirty-day morbidity and mortality after elective total shoulder arthroplasty: patient-based and surgical risk factors. J Shoulder Elbow Surg. 2015;24:24-30. 